\title{
Application of Denoising Methods of SINS Data Based on Wavelet Transform
}

\section{Efim Istomin}

Izhevsk State Technical University, ul. Studencheskaya 7, Izhevsk, 426069 Russia

Abstract: This paper is devoted to the application of data filtering methods based on wavelet transform (WT). The considered methods allow to restore noisy signals of inertial sensors in real time. A comparison of the discrete wavelet transform (DWT) and lifting wavelet transform (LWT) with different wavelet functions, decomposition levels and threshold filtering functions is presented.

Keywords: Discrete wavelet transform; real-time denoising; lifting scheme of wavelet transform.

\section{Introduction}

In the development of navigation systems, in particular SINS, the inertial sensors used in them often have a large measurement error and low signal-to-noise ratio. This leads to the fact that the interval of obtaining an acceptable solution of the SINS is limited to several seconds. To increase the stability of the navigation system and the accuracy of the obtained data, filtration should be used.

There are a lot of ways of digital signal processing: Kalman filters, Fourier transform, Wavelet transform, etc. In this paper two methods of filtering based on wavelet transforms are considered: DWT [1] and LWT [2] [3] [4].

\section{Methods Overview}

The basis of WT is the representation of the temporal signal in a frequency form. Due to this, it is possible to remove the noise component, while maintaining the main signal [5]. On account of this feature, the WT is widely used in the areas of information compression, filtering, highlighting of signal features, etc.

\subsection{Discrete wavelet transform}

Discrete wavelet transform [1] [5] is a mathematical algorithm intended for decomposition of a signal into a sequence of coefficients of small finite waves (wavelets).

Figure 1 shows the DWT scheme. The DWT consists of three steps: direct transformation, signal processing, inverse transformation.

The direct transformation consists in dividing the input signal $x[n]$ by the highand low-frequency components, due to the corresponding filters $\boldsymbol{h i d}[k]$ and $\operatorname{lod}[k]$ :

$$
\begin{aligned}
& d[k]=\sum_{n=-\infty}^{+\infty} x[n] \cdot \operatorname{hid}(-n+2 k) \\
& a[k]=\sum_{n=-\infty}^{+\infty} x[n] \cdot \operatorname{lod}(-n+2 k)
\end{aligned}
$$


Then the decimation of each second coefficient takes place, to preserve the length of the incoming signal.

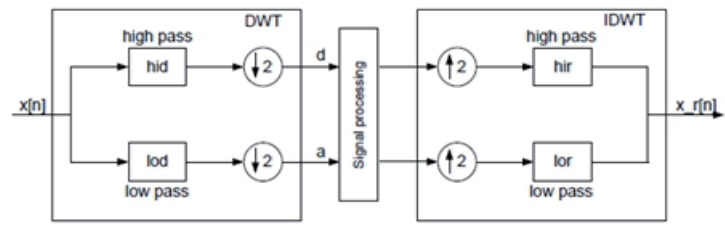

Fig. 1: Common scheme of discrete wavelet transform.

The output approximating $\alpha[k]$ and the detailing $d[k]$ coefficients are processed by threshold functions.

The inverse transformation consists in the reconstruction of the filtered signal, due to the coefficients $\operatorname{lor}[k]$ and $\operatorname{hir}[k]$ :

$x_{r[n]}=\sum_{n=-\infty}^{+\infty}(d[k] \cdot \operatorname{hir}(-n+2 k)+a[k] \cdot \operatorname{lor}(-n+2 k))$

To obtain more accurate frequency characteristics of the input signal, multi-level decomposition is used: the approximating coefficients are divided into high-frequency and low-frequency components. Reconstruction of such signal occurs in the reverse order of decomposition.

\subsection{Lifting wavelet transform}

Lifting wavelet transform - decomposition of the original signal into wavelets, due to the representation of DVP filters in the form of a finite sequence of elementary convolution operators.

Fig. 2 presents a common scheme of LWT. The scheme of direct lifting consists of three steps: split, predict, update. Consider the algorithm in details [6]:

Split - separation of the input signal into two components: even and odd samples.

Predict - obtaining a detailed coefficient (detail), by predicting an odd value with respect to the even value

$$
\text { Detail }=\text { Odd }- \text { Predict (Even) }
$$

Update - obtaining the coefficient of approximation (smooth), by updating the even value with respect to the detailed coefficient

$$
\text { Smoot }=\text { Even }- \text { Update }(\text { Detail })
$$

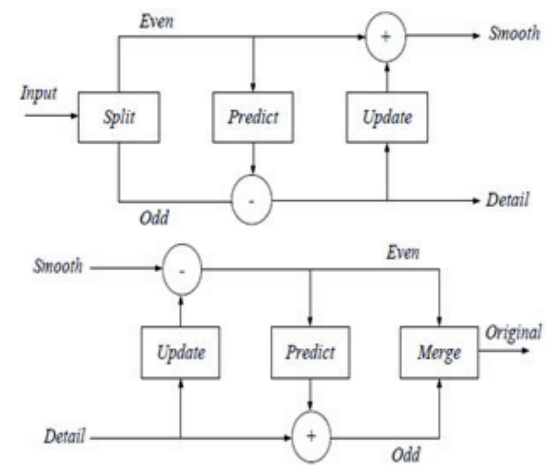

(a)

Fig. 2: Common scheme of lifting wavelet transform: (a) Forward wavelet transform. (b) Inverse wavelet transform.

The inverse transformation algorithm is mirrored to a direct transformation, so after decomposition an accurate reconstruction of the input signal is achieved:

$$
\begin{aligned}
& \text { Even }=\text { Smoot }- \text { Update }(\text { Detail }) \\
& \text { Odd }=\text { Detail }+ \text { Predict }(\text { Even })
\end{aligned}
$$

Merge - fusion of even and odd samples into one signal.

As in the case of DWT, each series of coefficients can be repeatedly decomposed into its components, obtaining more accurate frequency characteristics.

\subsection{Thresholding}

The original signal is filtered after calculation of approximating and detailed coefficients, i.e. after a direct wavelet transform. Noise reduction is achieved by applying a threshold function [7, 8]: the decomposition coefficient, that is less than absolute threshold value, becomes zero, otherwise it transforms. In this paper, following functions are considered: hard-thresholding

$O(t, \lambda)=\left\{\begin{array}{l}0, \text { if }|t| \leq \lambda \\ t, \text { if }|t|>\lambda\end{array}\right.$

soft-thresholding

$O(t, \lambda)=\left\{\begin{array}{l}t-\lambda, \text { if } d \geq \lambda \\ 0, \text { if }|d|<\lambda \\ t-\lambda, \text { if } d \leq-\lambda\end{array}\right.$

semisoft-thresholding

$O(t, \lambda)=\left\{\begin{array}{l}0, \text { if }|t|<\lambda \\ \operatorname{sign}(t) \sqrt{t^{2}+\lambda^{2}}, \text { if }|t| \geq \lambda\end{array}\right.$ 


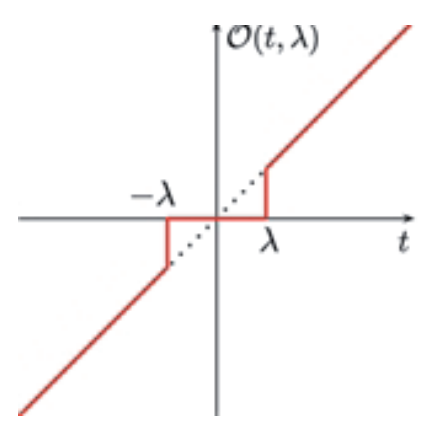

(a)
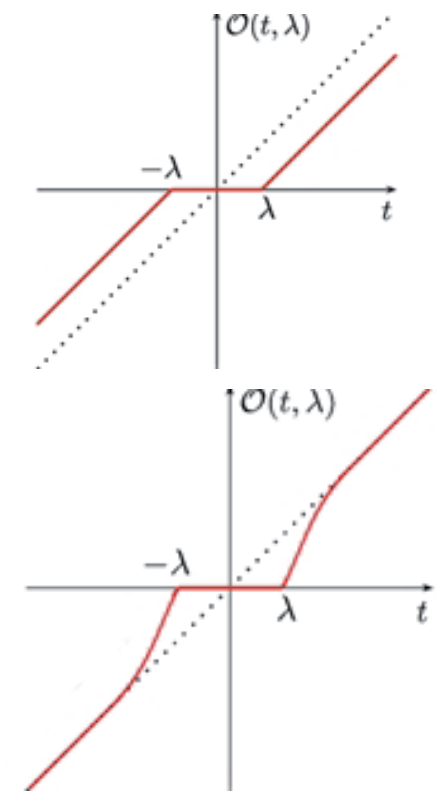

(c)

Fig. 3: Graphs of threshold functions: (a) Hard-thresholding. (b) Soft-thresholding. (c) Semisoft-thresholding.

\section{Experimental results}

In order to compare presented methods of noise reduction, evaluation criterion of denoising performance is introduced - root mean square error (RMSE):

$$
R M S E=\sqrt{\frac{1}{N} \sum_{i=0}^{N-1}\left(x_{i}-\hat{x}_{i}\right)^{2}}
$$

Where, $x_{i}$ is the original signal, $\hat{x}_{i}$ is the denoised signal, $\mathrm{N}$ is the length of the original signal.

The RMSE for noisy signals is 0.6.

\subsection{Discrete wavelet transform simulation}

The parameters of DWT are set as follows:

- wavelet function [5, 9]:

$\checkmark$ Symlet wavelet (sum2, sym4, sym8),

$\checkmark$ Daubechies wavelet ( db2, db4, db8),

$\checkmark$ Haar wavelet (Haar); decomposition level:

$\checkmark 2,4$;

- threshold function:

$\checkmark$ hard, soft, semi-soft.

A few denoising results of DWT are shown in Fig. 4.

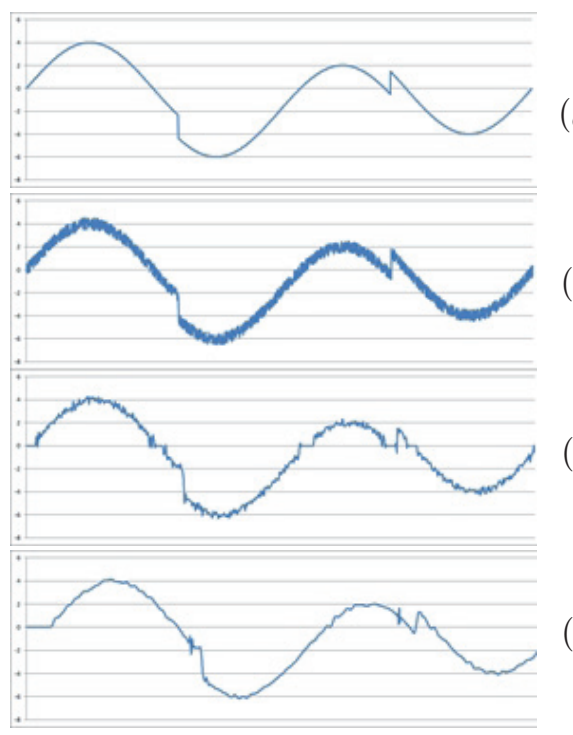

(a)

(b)

(c)

(d)

Fig. 4: Denoising results of discrete wavelet transform: (a) Original signal. (b) Noisy signal. (c) hard-thresholding denoising (wavelet db4, decomp. level 2). (d) hard-thresholding denoising (wavelet db4, decomp. level 4).

Denoising efficiency of DWT is presented in Tab. 1. The hard- and soft-threshold functions have best denoising performance. Application of the softthreshold function is more suitable for a high level of decomposition. The fourth decomposition level is more optimal, since the lag of denoised signal is increasing with growth of the level. The lag of denoised signal is described as follow:

$n_{\text {sample }}=\sum_{i=1}^{p}(N-1) \cdot 2^{i-1}$

Where, $N$ is the length of the wavelet filter, $p$ is the level of decomposition.

The wavelet type has a negligible impact on noise reduction effect, but there is a clear dependence of the filtering efficiency on the length of filter.

\subsection{Lifting wavelet transform simulation}

The parameters of LWT are set as follows:

- wavelet function:

$\checkmark$ Daubechies wavelet ( db2, db4, db8);

$\checkmark$ Width of moving window: 256 
Table 1: Results of denoising based on discrete wavelet transform.

\begin{tabular}{|l|l|l|l|l|l|l|}
\hline Thresholding func. & Hard & Soft & Semi. & Hard & Soft & Semi. \\
\hline Decomp. level & 2 & 2 & 2 & 4 & 4 & 4 \\
\hline haar & 0,406 & 0.666 & 0.415 & 0.183 & 0.389 & 0.197 \\
\hline $\mathrm{db} 2$ & 0.257 & 0,617 & 0.280 & 0.130 & 0,286 & 0,135 \\
\hline $\mathrm{db} 4$ & 0.215 & 0.546 & 0.239 & 0.124 & 0,244 & 0.125 \\
\hline $\mathrm{db} 8$ & 0.189 & 0.513 & 0.201 & 0.115 & 0.222 & 0.119 \\
\hline sym2 & 0.284 & 0.620 & 0.291 & 0.137 & 0.397 & 0.140 \\
\hline sym4 & 0.230 & 0.587 & 0.248 & 0.128 & 0.259 & 0.134 \\
\hline sym8 & 0.208 & 0.530 & 0.223 & 0.1230 & 0.243 & 0.125 \\
\hline
\end{tabular}

$\checkmark$ decomposition level: 2, 4;

$\checkmark$ threshold function: hard, soft, semi-soft.

A few denoising results of LWT are shown in Fig. 5.

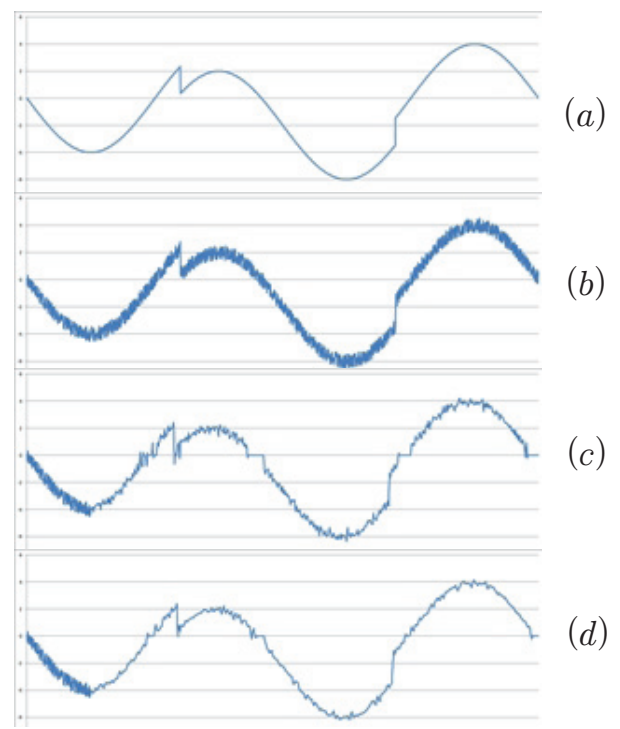

Fig. 5: Denoising results of lifting wavelet transform: (a) Original signal. (b) Noisy signal. (c) hard-thresholding denoising (wavelet db4, decomp. level 2, moving window width 256). (d) hard-thresholding denoising (wavelet db4, decomp. level 4, moving window width 256).

Denoising efficiency of LWT is presented in Tab. 2.

Table 2: Results of denoising based on lifting wavelet transform.

\begin{tabular}{|l|l|l|l|l|l|l|}
\hline $\begin{array}{l}\text { Thresholding } \\
\text { func. }\end{array}$ & Hard & Soft & Semi. & Hard & Soft & Semi. \\
\hline $\begin{array}{l}\text { Decomp. } \\
\text { level }\end{array}$ & 2 & 2 & 2 & 4 & 4 & 4 \\
\hline $\mathrm{db} 4$ & 0.267 & 0.603 & 0.297 & 0.144 & 0.281 & 0.146 \\
\hline $\mathrm{db6}$ & 0.210 & 0.571 & 0.233 & 0.130 & 0.247 & 0.135 \\
\hline
\end{tabular}

The LWT has similarities with DWT in signal denoising process. To eliminate the lag of denoised signal, a windowed LWT is used.

\section{Conclusions}

The application of the wavelet transform in inertial navigation is due to its noise reduction feature. This feature allows to obtain more appropriate data from inertial sensors. The threshold function has the greatest effect on the output signal. Use of the optimal algorithm for calculating threshold value will increase accuracy of the obtained data. The window modification of LWT provides acceptable denoising effect in real-time, unlike to DWT, which mathematical algorithm introduces a significant lag of the reconstructed signal.

\section{References and Notes}

[1] Mallat, S.G. (1989). A Theory for Multiresolution Signal Decomposition: The Wavelet Representation. IEEE Transactions on Pattern Analysis and Machine Intelligence

[2] Sweldens, W. (1998). The Lifting Scheme: A Construction of Second Generation Wavelets. SIAM Journal on Mathematical Analysis

[3] Zhaohua, L., Mi, Y., Mao, Y. (2014). An Improved Real-Time Denoising Method Based on Lifting Wavelet Transform. Measurement science review 14.3: 152-159

[4] Sweldens, W. (1996). The Lifting Scheme: A Custom-Design Construction of Biorthogonal Wavelets. Applied and Computational Harmonic Analysis

[5] Stark, H.G. (2005). Wavelets and Signal Processing: An Application-Based Introduction. N.p.

[6] Daubechies, I., Sweldens, W. (1998). Factoring Wavelet Transforms into Lifting Steps. Journal of Fourier Analysis and Applications

[7] Burhan, E. (2013). Comparison of Wavelet Types and Thresholding Methods on Wavelet Based Denoising of Heart 
Sounds. Journal of Signal and Information Processing

[8] Pivarčiová, E., Božek, P. Industrial production surety factor increasing by a system of fingerprint verification. ISEEE 2014; Sapporo City, Hokkaido; Japan; 26 April 2014 through 28 April 2014; Category number CFP1438X-PRT; Code 109001

[9] Dulam, S., Mittal, M. (2013). Implementation of Haar Wavelet through Lifting For Denoising. Int. Journal of Engineering Research and Applications 3.5: 1311-1314

[10] Frankovský, P., Hroncová, D., Delyová, I., Virgala, I. (2013). ). Modeling of dynamic systems in simulation environment MATLAB/Simulink-SimMechanics. American Journal of Mechanical Engineering, 1(7), 282-288

\section{Biographical notes}

Efim Istomin is a Ph.D.: student in mechatronics and robotics application at Izhevsk State Technical University (ISTU). He was born in 1992, in Izhevsk, Russia. He received the B.SC. and M.Sc degrees from ISTU in 2013 and 2015 respectively. His research interests are focused on inertial and integrated navigation systems.

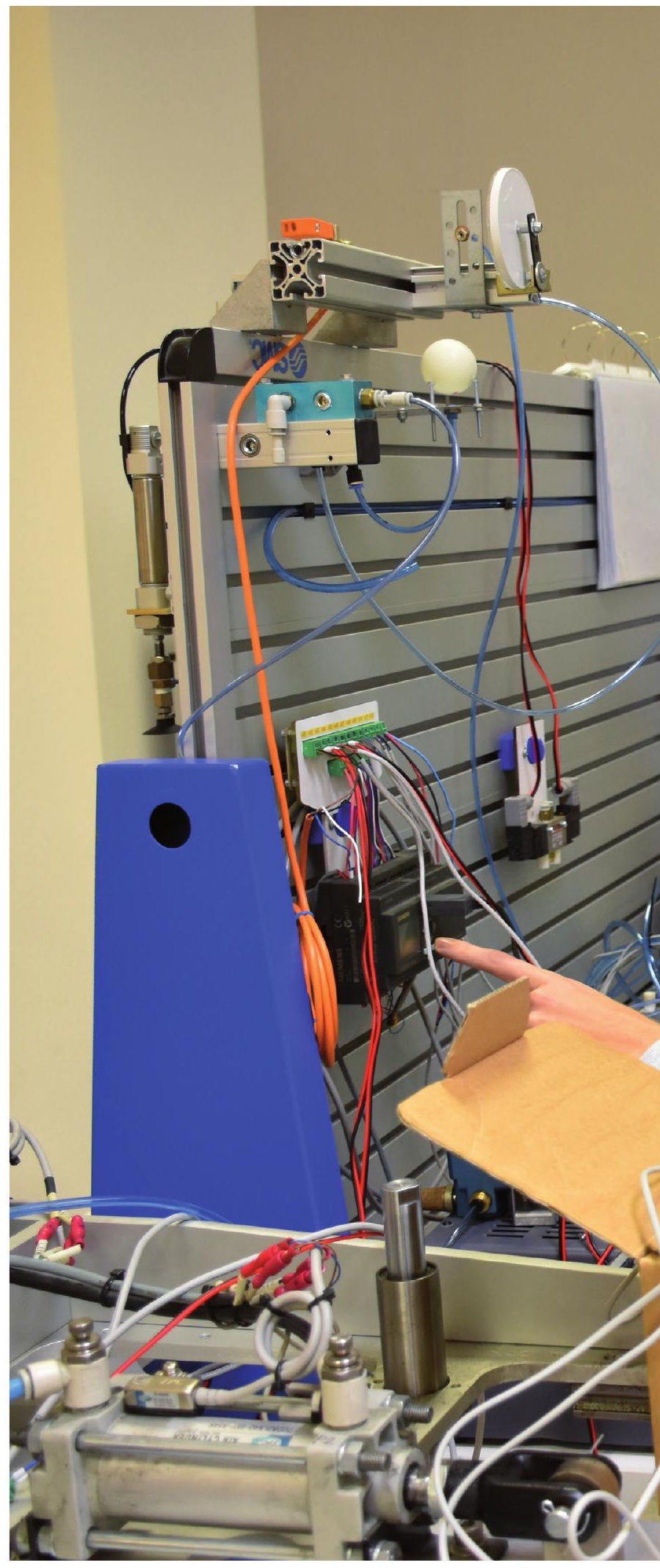

